some eukaryotic messengers, and in many laboratories any available mRNAs are being injected into these cells in the hope that they will be translated. It is hard to say what the failure rate is, but as Laskey, Gurdon and Crawford report in the current issue of the Proceedings of the National Academy of Sciences (69, 3665; 1972), the single-stranded RNA genome of encephalomyocarditis (EMC) virus, a picornavirus related to polio virus, can definitely be added to the list of successes.

After EMC RNA is injected into oocytes in the presence of $35 \mathrm{~S}$ methionine, polypeptides not present in oocytes injected with saline are made. As polyacrylamide gel electrophoretic analysis reveals these newly made polypeptides have identical mobilities to the EMC proteins made when the virus replicates in ascites cells. The fact that tryptic fingerprints of three of the polypeptides made in oocytes are identical to the fingerprints of the co-electrophoresing EMC proteins made in ascites cells leaves no room to doubt that in oocytes EMC RNA is not only extensively but also faithfully translated.

That EMC RNA is translated in Xenopus oocytes is not particularly surprising, but it is interesting that in these cells, as in ascites cells, which support virus replication, the polypeptide product of translation is processed after translation. There is good evidence that EMC RNA, like polio virus RNA, is translated, during virus replication in mammalian cells, into a single large precursor polypeptide that is subsequently cleaved to yield the mature viral proteins. These mature proteins can be detected in extracts of oocytes injected with EMC RNA so that it must be assumed that they arise by specific cleavage of the primary product of translation. This obviously implies that oocytes contain proteases with specificities similar to those in mammalian cells and that mechanisms for the post-translational cleavage of polypeptides may be widespread among eukaryotic cells. Whether EMC can be persuaded to replicate in oocytes to yield infectious progeny virions remains to be seen.

Laskey et al. point out the advantages of the oocyte system over cell-free systems from ascites cells but the latter have their adherents and uses. Oberg and Shatkin (ibid., 3589), for example, have used an ascites cell-free system programmed with EMC RNA to obtain further evidence for a unique initiation site for translation of this messenger. They fingerprinted the polypeptides made in the presence of $35 \mathrm{~S}$ MettRNA $_{\mathrm{M}}{ }^{\text {Met }}$ and 35S Met-tRNA ${ }_{\mathrm{F}}{ }^{\text {Met }}$; the latter tRNA translates only initiating AUG codons and fingerprints of poly. peptides made in its presence reveal a single $\mathbf{N}$ terminal tryptic peptide containing $35 \mathrm{~S}$ methionine. In other words, in this cell-free system, translation of EMC RNA and the RNAs of mouse Elberfeld virus and mengo virus is initiated at a unique site.

It remains true, of course, that compared with Escherichia coli cell-free systems for in vitro protein synthesis both ascites cell systems and the oocyte injection system are in their infancy, and one of the most remarkable results obtained of late with the $E$. coli system is that reported last April by Siegert et al. (ibid., 888). They found that in such a system the RNA of avian myeloblastosis virus is translated faithfully to yield four of the group specific antigens of the avian RNA tumour viruses. Gielkens, Salden and Bloemendal (FEBS Lett., 28, 348; 1972) now report that RNA from Rauscher leukaemia virus and mouse mammary tumour virus stimulates polypeptide synthesis in an $E$. coli cell-free system. Furthermore, at least some of the smaller polypeptides which are made co-electrophorese with proteins of the respective virions, and polypeptides specified by the leukaemia virus are not related to those specified by the mammary tumour virus. By contrast, globin messenger and calf lens crystallin messenger stimulate very little protein synthesis and the short peptides which are made are the products of aberrant initiation.

Gielkens et al. also mention that in collaboration with Gurdon they have injected Rauscher leukaemia virus RNA into oocytes, but they failed to detect evidence of its translation. It seems therefore that for RNA tumour virologists the $E$. coli cell-free systems hold great and unexpected interest.

\section{IMMUNOLOGY}

\section{Antigens and Ageing}

from our Cell Physiology Correspondent IT is always interesting when results emanating from different laboratories are diametrically opposed to one another, but when such conflicting reports are published in the same journal the reader finds it hard to know what to believe. In a recent issue of Experimental Cell Research two groups report on their studies of the fate of HLA antigens in ageing diploid cells; one group comes to the conclusion that these change with age whereas the other group believes that there is no change.

The HLA antigens have long been recognized as being useful for distinguishing between the tissues of genetically different individuals and it is also known that diploid fibroblasts retain the HLA antigens of the individual from whom they were derived. This fact is often taken to be an indication of the normality or non-neoplastic nature of the cells, just as the fact that the cells can only be subcultured a limited number of times, after which degenerative changes are evident, is thought to be akin to what is understood as ageing.

Sasportes and his colleagues (Nature, 233, 332; 1971) found that in some ten strains of fibroblasts obtained from skin biopsies, HLA antigens were lost as the cells grew older, the loss of antigens being evident before observable degenerative changes in the culture. Before this report appeared it had generally been accepted that histocompatibility antigens were retained throughout the life span of the cells, there being no change in the expression of HLA even during prolonged cultivation. Work

\title{
Aminoacylation of Viral RNAs
}

ONE of the most intriguing features of the single-stranded RNA genomes of plant viruses such as turnip yellow mosaic virus (TYMV) and tobacco mosaic virus (TMV) is the occurrence at the $3^{\prime}$ end of these RNAs of the sequence . . pCpCpA. This same $3^{\prime}$ terminal sequence is common to all transfer RNAs and, like transfer RNA molecules, the RNAs of TYMV and TMV can be esterified by an aminoacid. TYMV can be specifically charged with valine and TMV can, as Litvak et al. report in next Wednesday's Nature New Biology (January 17), be specifically charged with histidine using yeast histidyl-tRNA synthetase to catalyse the reaction. Furthermore, Litvak et al. have found that both histidyl TMV RNA and valyl TYMV RNA can under appropriate conditions react with the protein synthesis elongation factor 1 (EF1) purified from wheat and with
GTP to form a ternary complex of EF1.GTP.aminoacyl viral RNA. But TYMV RNA and TMV RNA that is not charged with an amino-acid does not react with EF1 and GTP.

Apparently the $3^{\prime}$ terminal sequence of these viral RNA genomes allows these RNA molecules to be acylated with an amino-acid and subsequently to form a ternary complex with an elongation factor. These events, as Litvak et al. speculate, may play a crucial part in regulating either the initiation of replication of these RNAs or the initiation of their translation by the protein synthesizing machinery of host plant cells ; for example, the complex of viral RNA with elongation factor may have a high affinity for ribosomes or, by analogy with the replication of phage $Q \beta$ RNA, elongation factor 1 may form part of a multimeric viral RNA replicase. 